\title{
Syntactic definitions of undefined: on defining the undefined
}

\author{
Zena Ariola ${ }^{1}$, Richard Kennaway ${ }^{2}$, Jan Willem Klop ${ }^{3}$, \\ Ronan Sleep ${ }^{4}$ and Fer-Jan de Vries ${ }^{5}$ \\ ${ }^{1}$ Computer and Information Science Department, University of Oregon, Eugene, Oregon \\ ${ }^{2,4}$ School of Information Systems, University of East Anglia, Norwich NR4 7TJ, UK \\ ${ }^{3,5}$ CWI, P.O. Box 94079, 1090 GB Amsterdam, the Netherlands \\ Contact addresses: ${ }^{5}$ ferjanषcwi.nl and ${ }^{2}$ jrk@sys.uea.ac.uk \\ ${ }^{2,4} \mathrm{fax}:+44603507720,{ }^{3,5} \mathrm{fax}:+31205924199$
}

\begin{abstract}
In the $\lambda$-calculus, there is a standard notion of what terms should be considered to be "undefined": the unsolvable terms. There are various equivalent characterisations of this property of terms. We attempt to find a similar notion for orthogonal term rewrite systems. We find that in general the properties of terms analogous to the various characterisations of solvability differ.

We give two axioms that a notion of undefinedness should satisfy, and explore some of their consequences. The axioms lead to a concept analogous to the Böhm trees of the $\lambda$-calculus. Each term has a unique Böhm tree, and the set of such trees forms a domain which provides a denotational semantics for the rewrite system. We consider several particular notions of undefinedness satisfying the axioms, and compare them.
\end{abstract}

AMS Subject Classification: $68 \mathrm{Q} 42$

CR Subject Classification: F1.1, F4.1, F4.2

Keywords \& Phrases: Orthogonal term rewriting systems, Böhm Trees, undefined terms, lambda calculus, solvability.

Note: All authors were partially sponsored by SEMAGRAPH II, ESPRIT working group 6345 . The second author was also partially supported by a SERC Advanced Fellowship, and by SERC Grant no. GR/F 91582.

\section{Introduction}

In the $\lambda$-calculus, there seems to be a well-established notion of what constitutes a "meaningless" or "undefined" term. The unsolvable terms are taken to be meaningless ([Bar84], 2.2.14) ${ }^{1}$.

A term $M$ is solvable if for its closure $M^{\prime}$ it holds that for all terms $\mathrm{P}$ there are terms $N_{1} \cdots N_{n}$ such that $M^{\prime} N_{1} \cdots N_{n}=P$. A term is unsolvable if it not solvable. Unsolvable terms can be characterized in various ways:

1. A term is unsolvable if and only if there is a reduction containing infinitely many head reductions (Wadsworth, cf. [Bar84], 8.3.11).

${ }^{1}$ Even for the $\lambda$-calculus the issue seems not be so clear either, as witnessed by [Bar92]. 
2. A term $M$ is solvable if and only it has a head normal form, which is a $\lambda$ expression of the form $\lambda x_{1} \cdots \lambda x_{n} . x E_{1} \cdots E_{k}$, where $x$ may be any of $x_{1} \cdots x_{n}$ or any other variable. (Wadsworth, cf. [Bar84], 8.3.14).

3. A term $t$ is unsolvable if and only if for every context $C[], C[t]$ has a normal form if and only if $C[s]$ has the same normal form for all $s^{2}$ ([Bar84], 14.3.24).

Evidence that unsolvability is a reasonable notion of undefinedness follows from:

1. All unsolvable terms can consistently be equated ([Bar84], 16.1.3).

2. The terms with no head normal form are exactly those which denote $\perp$ in graph model $P \omega$ of Plotkin and Scott ([Bar84], 19.1.10).

Barendregt defined the concept of Böhm tree with help of unsolvability, which led to the semantics of Böhm trees for $\lambda$-calculus ([Bar84], 18.3).

How much remains of this for term rewriting? What is a good concept of "undefined"? Clearly the $\lambda$-calculus definition of solvability does not carry over. The other characterizations do, although sometimes a bit modified.

This paper makes an initial attempt to identify certain classes of terms which are plausible candidates for the role of "undefined" or "meaningless" terms. Given a class of undefined terms satisfying some minimal axioms, the concept of Böhm tree arises naturally in the setting of infinitary term rewriting. From these axioms follow a general genericity lemma for term rewriting, similar to the genericity lemma in lambda calculus (cf. Proposition 14.3.24 in [Bar84]). As for $\lambda$-calculus the Böhm trees provide orthogonal term rewriting systems with denotational semantics, depending on the chosen set of undefined terms.

\section{Infinitary orthogonal term rewriting systems}

We will briefly recall infinitary orthogonal term rewriting systems involving both finite and infinite terms. For details of finitary term rewriting the reader is referred to [vL90] and [AGM92] and for an account of infinitary term rewriting to [KKSdV93] or the papers of Kennaway et al. in [SPvE93].

An infinitary term rewriting system (TRS, usually this abbreviation is reserved for the finitary term rewriting systems only) over a signature $\Sigma$ is a pair $\left(\operatorname{Ter}^{\infty}(\Sigma), R\right)$ consisting of the set $\operatorname{Ter}^{\infty}(\Sigma)$ of finite and infinite terms over the signature $\Sigma$ and a set of rewrite rules $R \subseteq \operatorname{Ter}(\Sigma) \times \operatorname{Ter}^{\infty}(\Sigma)$. If all function symbols of $\Sigma$ occur in $R$ we will write just $R$ for $\left(T e r^{\infty}(\Sigma), R\right)$.

The set $\operatorname{Ter}^{\infty}(\Sigma)$ is the metric completion of the set of $\operatorname{Ter}(\Sigma)$ of finite terms with the metric: $d: \operatorname{Ter}(\Sigma) \times \operatorname{Ter}(\Sigma) \rightarrow[0,1]$. The distance $d(t, s)$ of two terms $t$ and $s$ is 0 , if $t$ and $s$ are equal, and $2^{-k}$, otherwise, where $k \in \omega$ is the largest natural

\footnotetext{
${ }^{2}$ Actually in [Bar84] is proved that: a term $t$ is unsolvable if for every context $C[]$ it holds that $C[t]$ has a normal form implies $C[s]$ has the same normal form for all $s$.

The second implication can clearly be reversed (take $s=t$ ). The reverse of the first implication says that if $t$ is solvable, then there is a context $C[]$, a normal form $r$, and a term $s$ such that $C[t]=r$ and $C[s] \neq r$. But this is surely true - take s to be some other solvable term and use separability to construct a context $C[]$ such that $C[t]$ and $C[s]$ have different normal forms.
} 
number such that all nodes of $s$ and $t$ at depth less than or equal to $k$ are equally labeled.

Substitutions, contexts and reduction steps generalize trivially to the set of infinitary terms $\operatorname{Ter}^{\infty}(\Sigma)$.

A rewrite rule $l \rightarrow r$ is left-linear if no variable occurs more than once in the left-hand side $l . R$ is non-overlapping if for any two left-hand sides $s$ and $t$, any position $u$ in $t$, and any substitutions $\sigma$ and $\tau: \operatorname{Var} \rightarrow \operatorname{Ter}^{\infty}(\Sigma)$ it holds that if $(t / u)^{\sigma}=s^{\tau}$ then either $t / u$ is a variable or $t$ and $s$ are left-hand sides of the same rewrite rule and $u=\lambda$ (i.e. non-variable parts of different rewrite rules don't overlap and non-variable parts of the same rewrite rule overlap only entirely). A (in)finitary term rewriting system $R$ is orthogonal if its rules are left-linear and non-overlapping.

A transfinite reduction sequence consists of a function $f$ whose domain is an ordinal $\alpha$, such that $f$ maps each $\beta<\alpha$ to a reduction step $f_{\beta} \rightarrow f_{\beta+1} . f$ is Cauchy continuous if the sequence of terms $\left\{f_{\beta} \mid \beta<\alpha\right\}$ is a continuous function from $\alpha$ (with the usual topology on ordinals) to $\operatorname{Ter}^{\infty}(\Sigma)$ (with the metric topology). For each $\beta<\alpha$, let $d_{\beta}$ be the depth of the redex reduced in the step from $f_{\beta}$ to $f_{\beta+1}$. The sequence is strongly continuous if for every limit ordinal $\lambda<\alpha$, the sequence $\left\{d_{\beta} \mid \beta<\lambda\right\}$ tends to infinity. It is Cauchy convergent if it is Cauchy continuous and converges topologically to a limit, denoted by $f_{\alpha}$. It is strongly convergent if in addition the sequence $\left\{d_{\beta} \mid \beta<\alpha\right\}$ tends to infinity. As we have argued in [KKSdV93], strongly convergent reduction sequences are the appropriate notion of transfinite reduction sequence, as Cauchy convergence alone is insufficient to allow the definition of the fundamental notions of residuals, compression and (projection or) strip lemma.

We write $t \rightarrow_{\alpha} s$ (resp. $t \rightarrow_{\leq \alpha} s$ ) to denote a strongly converging reduction of length $\alpha$ (resp. at most $\alpha$ ) starting from $\alpha$ and converging to $\beta$, and $t \rightarrow^{\infty} s$ for a strongly converging reduction of any finite or infinite length. $t \rightarrow^{*} s$ denotes a reduction of finite length (including zero). Consider some examples:

1. Rule $A(x, y) \rightarrow A(y, x)$, sequence $A(B, C) \rightarrow A(C, B) \rightarrow A(B, C) \rightarrow A(C, B) \cdots$

2. Rule $A(x, y) \rightarrow A(y, x)$, sequence $A(D, D) \rightarrow A(D, D) \rightarrow A(D, D) \rightarrow A(D, D) \cdots$

3. Rule $C \rightarrow S(C)$, sequence $C \rightarrow S(C) \rightarrow S(S(C)) \rightarrow \cdots S(S(S(\cdots)))$.

Example (1) is a diverging reduction sequence. Example (2) is Cauchy convergent with limit $A(D, D)$. Example (3). is strongly convergent with limit $S^{\omega}$ (i.e. $S(S(S(\ldots))))$.

In order to transfer certain theorems about finitary orthogonal term rewriting to the infinitary setting we need to extend the definition of descendant to account for what happens at limit points. For a set of positions $v$ of $t_{0}$ and a reduction sequence $\alpha$ from $t_{0} \rightarrow_{\alpha} t_{\alpha}$, the set $v \backslash \alpha$ of descendants of $v$ by $t_{0} \rightarrow_{\alpha} t_{\alpha}$ in $t_{\alpha}$ is defined by induction on the ordinal $\alpha$. When $\alpha$ is finite, this is the standard notion. If $\alpha$ is a limit ordinal, then $v \backslash \alpha$ is defined in terms of the sets $v \backslash \beta$ for all $\beta<\alpha$, as follows: $u \in v \backslash \alpha$ if and only if $\exists \beta<\alpha \forall \gamma(\beta<\gamma<\alpha \rightarrow u \in v \backslash \gamma)$

Lemma 1. Strip Lemma. Let $t_{0} \rightarrow_{\alpha} t_{\alpha}$ be a strongly converging reduction of $t_{0}$ to $t_{\alpha}$ and let $t_{0} \rightarrow s_{0}$ be a reduction of a redex $R$ of $t_{0}$. Then there is a strongly converging reduction $s_{0} \rightarrow_{\beta} s_{\alpha}$ consisting of a concatenation of strongly converging reductions $s_{\gamma} \rightarrow \beta_{\gamma} s_{\gamma+1}$ for $\gamma<\alpha$, where for all $\gamma \leq \alpha, s_{\gamma}$ is obtained by contraction of all 
descendants of $R$ in $t_{\gamma}$ and $s_{\gamma} \rightarrow_{\beta_{\gamma}} s_{\gamma+1}$ is a strongly converging reduction of all descendants of the contracted redex in $t_{\gamma .} \rightarrow t_{\gamma+1}$.

The notion of Lévy equivalence can be generalised to the infinitary context. The compression lemma then states that for any strongly converging reduction there exists a Lévy equivalent strongly converging reduction of length at most $\omega$. For the present paper the following version suffices:

Lemma 2. Simple Compression Lemma. If $t \rightarrow^{\infty} s$ then $t \rightarrow \leq \omega s$.

In infinitary term rewriting the transfinite Church-Rosser property (whenever $t_{1} \leftarrow_{\alpha} t \rightarrow_{\beta} t_{2}$ there exists a term $s$ such that $t_{1} \rightarrow_{\gamma} s \leftarrow_{\delta} t_{2}$ ) holds only for almost non-collapsing orthogonal TRSs. A TRS is almost non-collapsing if it has at most one rule whose right hand side is a single variable, in which case the corresponding left hand side contains no other variables. A counterexample is given by the rules $C \rightarrow A(B(C)), A(x) \rightarrow x, B(x) \rightarrow x$. The term $C$ can strongly converge to both $A^{\omega}$ and $B^{\omega}$.

In the rest of this paper all reductions will be assumed to be potentially infinite, strongly converging reductions.

\section{Axioms for undefined terms}

There are two properties which we consider any notion of undefinedness should satisfy, which we state here as two axioms on the set $U$ of undefined terms.

Firstly, evaluation of an undefined term should not yield a defined term (otherwise the original term would be considered to be defined). Conversely, evaluation of a defined term should not yield an undefined term. This assumption depends on the fact that we are dealing only with orthogonal term rewrite systems. In other systems, a term might reduce to both an undefined term and to a defined term, and it is less clear how to classify such a term.

Axiom 1. $U$ and its complement are closed under strongly converging reduction.

Secondly, terms without root stable form should immediately be classified as undefined.

Definition 3. A term is root stable if it cannot be reduced to a redex. $t$ has a root stable form if it can be reduced to a root stable term $s$. $s$ is said to be a root stable form of $t$.

Intuitively, if we can reduce a term to a root-stable form, then the information at the root embodies part of the total information obtainable from the term. If a term has no root-stable form, then it contains no information, and should be considered to be undefined. Hence:

Axiom 2. $U$ contains every term which has no root stable form. 
It is convenient to use the symbol $\perp$ to denote undefinedness. We add this to the signature as a nullary function symbol. Terms possibly containing $\perp$ are called partial terms. $\perp$ is conventionally defined to be not root-stable. A partial order is defined on partial terms by requiring that $\perp \subseteq t$ for every term $t$, and that every function symbol is monotonic. If $s$ $t$, then $s$ is said to be a prefix of $t$.

The next definition extends to partial terms the classification of terms into defined and undefined.

Definition 4. $U_{\perp}=\left\{t \in \operatorname{Ter}^{\infty}(\Sigma \cup\{\perp\}) \mid \exists s \in U . t \sqsubseteq s\right\}$.

For the remaining definitions and theorems, we assume that $U$ satisfies axioms 1 and 2. All terms considered are partial terms.

Lemma 5. $U$ is closed under reduction if and only if $U_{\perp}$ is.

Proof. "If" is trivial. For the reverse direction, let $t \in U_{\perp}$. Take a variable $x$ not occurring in $t$ and let $t^{\prime}$ be obtained from $t$ by replacing every occurrence of $\perp$ by $x$. Then every reduction of $t$ corresponds to a reduction of $t^{\prime}$. If $t$ were reduced to a term outside $U_{\perp}$, the corresponding reduction of $t^{\prime}$ would lead to a term outside $U$.

This implies that $U$ satisfies axiom 1 if and only if $U_{\perp}$ does.

Definition 6. $U$-reduction (notated $\rightarrow U$ ) is the union of the reduction relation of the given system with the rule $t \rightarrow U \perp$ for all $t \in U_{\perp}$. A normal form with respect to this relation is, by analogy with lambda calculus, called a $B o ̈ h m_{U}$ tree or $B o ̈ h m_{U}$ normal form.

The following theorems establish some basic properties of Böhm $U$ normal forms, and flesh out the intuition that undefined terms are not "visible" to any context in which they are placed. Preliminary to this, we need some properties of prefixes and root-stable terms.

Lemma 7. Let $t \rightarrow^{\infty} s$, and let $r$ be a finite prefix of $s$. Then there is a term $q$, also having $r$ as a prefix, such that $t \rightarrow^{*} q$.

Proof. By the Compression Lemma, $t \rightarrow \leq \omega \mathrm{s}$. By strong convergence, every term in this sequence from some point before the limit onwards has $r$ as a prefix.

Lemma 8. 1. The set of root stable terms is closed under strongly converging reductions.

2. The set of terms having a root stable form is closed under strongly converging reduction.

Proof. 1. Immediate from the definition.

2. This follows from the first item and theorem 18, a general theorem that we will prove in the next section. 
Lemma 9. If a term can be reduced to root-stable form, it can be reduced to rootstable form in finitely many steps.

Proof. Let $t$ be reducible to a root-stable term $s$. By the Compression Lemma, this can be done in at most $\omega$ steps. By strong convergence, the reduction of $t$ to $s$ must have the form $t \rightarrow^{*} r \rightarrow^{\omega} s$, where $r \rightarrow^{\omega} s$ performs no root reductions. Suppose that $r$ is not root-stable. Then $r$ can be reduced to a redex, and by lemma 7 can be reduced to a redex in finitely many steps. Let $r \rightarrow^{*} q$ be such a reduction. Now apply the Strip Lemma to the sequences $r \rightarrow^{\omega} s$ and $r \rightarrow^{*} q$, to obtain sequences $s \rightarrow^{\infty} p$ and $r \rightarrow^{\infty} p$. Neither of the given sequences contains any root reductions, therefore neither do the sequences constructed by the Strip Lemma. $r$ is a redex, so by orthogonality, $p$ must also be a redex, contradicting the root-stability of $s$.

Theorem 10. Every term has a unique Böhm $m_{U}$ tree.

Proof. Define $t$ to be stable to depth $n$ if for every occurrence $u$ of $t$ of length at most $n, t \mid u$ is either $\perp$ or root-stable.

Let $t$ be any term. If $t$ has no root-stable form, then by the first axiom, $t \rightarrow U \perp$. By lemma 8, the set of such terms is closed under reduction, so $\perp$ is the only $\operatorname{Böhm}_{U}$ tree which $t$ can reduce to. Otherwise, $t$ reduces, and by lemma 9 in finitely many steps, to a root-stable term $s$. The finite Church-Rosser property (of the ordinary reduction rules) implies that the root symbol or root variable of $s$ is determined uniquely. Therefore every term can be $U$-reduced to a term which is stable to depth 1 , and its root symbol, whether $\perp$, a variable, or a function symbol, is unique.

Let $t$ be stable to depth $n$. For any occurrence $u$ of $t$ of length $n, t \mid u$ can be reduced in finitely many steps to a term stable to depth 1 . Doing this for all such occurrences gives a finite reduction of $t$ to a term stable to depth $n+1$. Furthermore, the prefix of this term down to depth $n+1$ is uniquely determined.

Repeating indefinitely gives a strongly convergent $U$-reduction of $t$ to a unique term stable to all finite depths, i.e. a Böhm ${ }_{U}$ tree.

Definition 11. $\mathcal{B}_{U}(t)$ denotes the $\mathrm{Böhm}_{U}$ tree of $t$.

Theorem 12. For any term $t$, and any finite term $s \sqsubseteq \mathcal{B}_{U}(t)$, there is a finite reduction of $t$ to some term $r$ such that $s \sqsubseteq r$.

Proof. In the proof of theorem 10 we constructed for each term a strongly converging reduction to $\mathrm{Böhm}_{U}$ normal form of length at most $\omega$. By the definition of strong convergence any finite prefix of the final term is present at some finite stage during the reduction.

Theorem 13. For any term $t, t \in U_{\perp}$ if and only if $\mathcal{B}_{U}(t)=\perp$.

Proof. $\Rightarrow$ : immediate from the definition of $U$-reduction.

$\Leftarrow$ : From the definition of $U$-reduction, the final step of a $U$-reduction of $t$ to $\perp$ must have the form $s \rightarrow \perp$, where $s \in U_{\perp}$. Since by axiom 1 the complement of $U$ is closed under reduction, $t$ must also be in $U_{\perp}$.

Theorem 14. If $s \sqsubseteq t$ then $\mathcal{B}_{U}(s) \sqsubseteq \mathcal{B}_{U}(t)$. 
Proof. By induction on a $U$-reduction of $s$ to $\mathcal{B}_{U}(s)$. It is immediate from the definition of $U$-reduction that $t$ has a $U$-redex everywhere that $s$ does. Therefore if $s \rightarrow U s^{\prime}$ by a reduction at occurrence $u$, then for some $t^{\prime}, t \rightarrow t^{\prime}$ by reduction at $u$, and $s^{\prime} \sqsubseteq t^{\prime}$. By continuity, it follows that there is a term $t^{\prime}$ such that $t \rightarrow \infty t^{\prime}$ and $\mathcal{B}_{U}(s) \sqsubseteq t^{\prime}$. Therefore $\mathcal{B}_{U}(s) \sqsubseteq \mathcal{B}_{U}\left(t^{\prime}\right)=\mathcal{B}_{U}(t)$.

Theorem 15. For any terms $s$ and $t, \mathcal{B}_{U}(s) \sqsubseteq \mathcal{B}_{U}(t)$ if and only if for every context $C[], \mathcal{B}_{U}(C[s]) \sqsubseteq \mathcal{B}_{U}(C[t])$.

Proof. $\Leftarrow$ : immediate by taking $C[]=[]$.

$\Rightarrow$ : By uniqueness of Böhm ${ }_{U}$-normal forms (theorem 10), the right hand side is equivalent to $\mathcal{B}_{U}\left(C\left[\mathcal{B}_{U}(s)\right]\right) \sqsubseteq \mathcal{B}_{U}\left(C\left[\mathcal{B}_{U}(t)\right]\right)$. In other words, it is sufficient to prove the theorem in the case where $s$ and $t$ are Böhm normal forms. When this is the case, the left hand side is equivalent to $s$ being a prefix of $t$. This implies that $C[s] \sqsubseteq C[t]$. By theorem 14 the right hand side follows.

Theorem 16. For any term $t, t$ is in $U_{\perp}$ if and only if for any context $C[]$ and term $s, \mathcal{B}_{U}(C[t]) \sqsubseteq \mathcal{B}_{U}(C[s])$.

Proof. $\Leftarrow$ : Take $C[]=[]$ and $s=\perp$. Then the right hand side says that $\mathcal{B}_{U}(t)=1$. By theorem 13, $t \in U_{\perp}$.

$\Rightarrow$ : Let $t \in U_{\perp}$. By theorem $13, \mathcal{B}_{U}(t)=\perp$. Therefore $\mathcal{B}_{U}(C[t])=\mathcal{B}_{U}(C[\perp])$. $\perp \sqsubseteq s$, so by theorem $15, \mathcal{B}_{U}(C[\perp]) \sqsubseteq \mathcal{B}_{U}(C[s])$.

This theorem is a generalization of the genericity lemma occuring in lambda calculus (cf. Proposition 14.3.24 in [Bar84]).

Definition 17. A term is totally defined if none of its subterms (including the term itself) is in $U_{\perp}$. (Note that such a term necessarily cannot contain 1 .)

We note that our axioms are expressed in terminology which applies to the lambda calculus as well. The set of unsolvable terms of lambda calculus satisfies all the above axioms and theorems, as do the sets of easy terms and the terms of order 0 [Bar92].

\section{Candidates for syntactic definitions of undefinedness}

In this section we describe four- different notions of undefinedness. For each one, we state which of the axioms of the previous section it does or does not satisfy. In addition, with each definition there is associated a set of "certainly-meaningful" terms; with these we can state stronger versions of some of the axioms.

We can simplify the task of establishing that the various concepts satisfy the axioms for undefinedness, by the following theorem.

Theorem 18. Let $S$ be a set of terms having the following two properties:

1. $S$ is closed under transfinite reduction.

2. For every term $t$, if there is an $s \in S$ such that $t \rightarrow^{\infty} s$, then there is an $s^{\prime} \in S$ such that $t \rightarrow{ }^{*} s^{\prime}$. 
Let $\bar{S}$ be the set of all terms $t$ such that there is an $s \in S$ for which $t \rightarrow^{\infty} s$. Then $\bar{S}$ is closed under transfinite reduction.

Proof. Let $t \in \bar{S}$. That is $t \rightarrow^{\infty} p$ for some $p \in S$. Suppose that $t \rightarrow^{\infty} s$. We must show that $s \in \bar{S}$. From $t \in \bar{S}$ it follows that $t \rightarrow^{\infty} p$ for some $p \in S$. Hence, by condition 2, there is an $r \in S$ such that $t \rightarrow^{*} r$. By the Strip Lemma, there must exist a $q$ and reduction sequences $s \rightarrow^{\infty} q$ and $r \rightarrow^{\infty} q$. By condition $1, q \in S$. Therefore $s \in \bar{S}$.

\subsection{Transparent and opaque terms}

Definition 19. A closed term is a transparent value if it has the form $\sigma(l \mid u)$, where $l$ is the left-hand side of a rule, $u$ is a position in $l$ such that $l \mid u$ is not a variable, and $\sigma$ is a substitution. An open term is a transparent value if some closed instance is. A term is transparent if it can be reduced to a transparent value; otherwise, it is opaque. $U_{0}$ is the set of opaque terms. A totally transparent value is a term, all of whose subterms are transparent values.

For example in a TRS expressing basic arithmetic one might encounter the rules:

$$
\begin{aligned}
& \operatorname{Add}(0, y) \rightarrow y \\
& \operatorname{Add}(S(x), y) \rightarrow S(\operatorname{Add}(x, y))
\end{aligned}
$$

In this fragment the terms $0, S(0)$ and $A d d(S(x), y)$ are examples of transparent values. Terms like $A d d(x$, True) and $S($ True $)$ are opaque.

The intuition behind the definition of $U_{o}$ is that for a term to be meaningful, it must be possible for it to be pattern-matched from outside.

Note that while the sets of opaque and transparent terms are in general not recursive, the sets of transparent values and totally transparent values are. Transparent values can be thought of as "obviously meaningful" terms. Totally transparent values consist entirely of obviously meaningful components.

The concept of transparent value can be regarded as a generalisation to arbitrary orthogonal rewrite systems of the notion of constructor term. A constructor system is a TRS in which every function symbol is either an operator, i.e. appears at the root of at least one left-hand side, and does not appear anywhere else in any left-hand side, or a constructor, i.e. a symbol which does not appear at the root of any left-hand side. A constructor term is one having a constructor symbol at its root. It is clear that in a constructor system, every transparent value is a constructor term. In practical examples of constructor systems, such as programs in most functional languages, one typically also finds that every term with a sufficiently large prefix consisting entirely of constructors is a transparent value. In fact, if constructor symbols always arise in conjunction with operators whose rules discriminate on the constructor, then the transparent values will be precisely the constructor terms.

Lemma 20. The set of transparent values is closed under transfinite reduction. 
Proof. Let $t=\sigma(l \mid u)$, let $l$ be the left hand side of some rule, and let $u \neq\langle\rangle$ be a position of a function symbol in $l$. Let $t \rightarrow^{\infty} \mathrm{s}$. We prove by induction on the length of the sequence that $s$ is an instance of $l \mid u$.

Let $t \rightarrow s$ in one step, by reduction of a redex at position $v$. By orthogonality, $u \cdot v$ cannot be a position of a function symbol in $l$, since otherwise $\sigma(l)$ would have conflicting redexes at \langle\rangle and at $u \cdot v$. Therefore $u \cdot v$ is an extension of a position of a variable $x$ in $l$, and $s$ is an instance $\sigma^{\prime}(l \mid u)$, where $\sigma^{\prime}$ differs from $\sigma$ only at $x$.

Suppose $t_{0} \rightarrow_{\alpha} t_{\alpha}$, where $t_{0}=\sigma(l \mid u)$ is transparent, $\sigma, l$, and $u$ being as before. Assume by induction that each term $t_{\beta}$ for $\beta<\alpha$ is an instance of $l \mid u$. Since $l \mid u$ is finite, convergence implies that $t_{\alpha}$ is also an instance of $l \mid u$, and hence is a transparent value.

Lemma 21. If $t$ can be reduced to a transparent value, it can be reduced to one in finitely many steps.

Proof. Suppose $t$ can be reduced to an instance of a term $l \mid u$, where $l$ is a left hand side and $u$ is a nonempty position of a function symbol in $l$. Since $l \mid u$ is finite, by lemma $7, t$ can be reduced to such a term in finitely many steps.

By theorem 18 we obtain from these lemmas:

Corollary 22. The set of transparent terms is closed under transfinite reduction.

Theorem 23. In an orthogonal TRS:

1. $U_{o}$ satisfies axioms 1 and 2.

2. Every transparent value is root stable.

3. Every totally transparent value is a normal form.

Proof. 1. By orthogonality $U_{o}$ is trivially closed under reduction, and by corollary 22 so is its complement. From or thogonality it is immediate that $U_{o}$ contains all terms without root stable form.

2. Immediate from orthogonality.

3. Immediate from the previous item.

However, note that in general not all normal forms are transparent. An example is given by a term such as $A d d$ (True, True), given a set of rules for $A d d$ which require both arguments to be integers. This term is an opaque normal form.

\section{$4.2 \omega$-reduction}

$\omega$-reduction is based on the notion that in general, one cannot discover the normal form of a term other than by reducing it to normal form. The information about the normal form of a term that we can discover without performing any reduction may be approximated by imagining that every redex is undefined, and that every term that might possibly be a redex, given that nothing is known about its subterms which are redexes, is also undefined. 
Definition 24. Let $\omega$ be a new nullary function symbol. Define a partial ordering on terms:

1. $\omega \leq t$ for all $t$

2. $F\left(t_{1}, \ldots, t_{n}\right) \leq F\left(t_{1}^{\prime}, \ldots, t_{n}^{\prime}\right)$ if $t_{i} \leq t_{i}^{\prime}$ when $1 \leq i \leq n$.

If $s \leq t$ then $s$ is an $\omega$-prefix of $t$. $\omega$-reduction is defined by the rule $t \rightarrow \omega$ if $t \leq s$ for some redex $s$.

Proposition 25. $\omega$-reduction is confluent and strongly normalising (even if the TRS is not orthogonal).

Definition 26. $\omega_{n f}(t)$ is the (existing and unique, by the previous proposition) $\omega$ normal form of $t$. $t$ is an $\omega$-value if there is a finite $\omega$-prefix $s$ of $t$ such that $\omega_{n f}(s) \neq \omega$. $t$ is $\omega$-defined if it reduces to an $\omega$-value, otherwise it is $\omega$-undefined. $U_{\omega}$ is the set of $\omega$-undefined terms. A total $\omega$-value is a term, all of whose subterms are $\omega$-values.

For finite terms, the above definition of $t$ being an $\omega$-value is equivalent to the $\omega$-normal form of $t$ not being $\omega$. For infinite terms, this is not the case. For example, given a rule whose left hand side is $F(A)$, the infinite term $F(F(F(\ldots)))$ is a normal form, hence also an $\omega$-normal form, but every finite $\omega$-prefix $\omega$-reduces to $\omega$. The more complicated definition of $\omega$-value is necessary to ensure that the $\omega$-undefined terms are closed under reduction.

Lemma 27. The set of $\omega$-values is closed under transfinite reduction.

Proof. Let $t$ be a $\omega$-value, with $\omega$-normal form $s$. Then for every position $u$ of a proper function symbol in $s, u$ cannot be a position of a redex in any term which is an instance of $s \mid u$. Therefore $s$ is a prefix of every term which $t$ reduces to, and since $s$ is a $\omega$-normal form, it is a prefix of the $\omega$-normal form of every such term.

Lemma 28. If a term can be reduced to a $\omega$-value, it can be reduced to an $\omega$-value in finitely many steps.

Proof. Let $t$ be reducible to a $\omega$-value $s$. That property of $s$ depends only on some finite prefix $s^{\prime}$ of $s$ in $\omega$-normal form. By lemma $7, t$ is reducible to a term having $s^{\prime}$ as a prefix in finitely many steps. Such a term is a $\omega$-value.

By theorem 18 we now obtain from these lemmas:

Corollary 29. The set of terms having a $\omega$-normal form is closed under strongly converging reduction.

Theorem 30. In an orthogonal TRS:

1. $U_{\omega}$ satisfies axioms 1 and 2.

2. Every $\omega$-value is root stable.

3. The total $\omega$-values are the normal forms. 
Proof. 1. The $\omega$-undefined terms are closed under reduction because they are a class of terms not reducible to members of a certain class. By corollary 29, the complement of $U_{\omega}$ is closed under strongly converging reduction. It is immediate that $U_{\omega}$ contains all terms without root-stable form.

2. Immediate.

3. Immediate from the previous item.

\subsection{Root stable form}

In earlier sections we defined the notion of root stable form. This itself gives rise to a minimal notion of undefinedness, in which the "meaningless" terms are taken to be exactly the terms without root stable form.

Let $U_{r s}$ be the set of terms without root stable form. By analogy with the notions of transparent value and $\omega$-value, we might call root-stable terms rs-values. The total $r s$-values would then be the terms, all of whose subterms are root-stable, i.e. the normal forms.

Theorem 31. In an orthogonal TRS:

1. $U_{r s}$ satisfies axioms 1 and 2.

2. The following are equivalent for any term $t$ :

(a) $t$ is a normal form.

(b) $t$ is a total rs-value.

(c) Every subterm of $t$ is root stable.

Proof. 1. $U_{r s}$ is trivially closed under transfinite reduction. Its complement is closed, by corollary 8 . The second axiom is trivial from the definition.

2. Trivial.

\subsection{Hypercollapsing terms}

A collapsing rule is a rewrite rule whose right hand side is a variable. A collapsing redex is a redex by such a rule. A hypercollapsing term is a term from which there is a (strongly continuous) reduction sequence containing infinitely many collapsing reduction steps at the root.

The notion of hypercollapsing terms as being the undefined terms only satisfies the first axiom for undefinedness. We include it here because the hypercollapsing terms play a key role in the failure of the Church-Rosser property for infinitary rewriting in orthogonal TRSs. In [KKSdV93] we have shown that in general, the Church-Rosser property holds only up to the equivalence of hypercollapsing terms.

Let $U_{h c}$ be the class of hypercollapsing terms.

Theorem 32. In an orthogonal TRS:

1. $U_{h c}$ satisfies axiom 1.

2. A normal form contains no hypercollapsing subterms. 
Proof. 1. In [KKSdV93] we have proved that hc-terms are closed under reduction. If a term reduces to a hypercollapsing term, then it is clearly a hypercollapsing term itself. Hence the complement of $U_{h c}$ is closed under reduction as well.

2. Trivial.

However,

1. $U_{h c}$ in general does not satisfy axiom 2. Consider the rule $A \rightarrow A$ and the term $A$. This term has no root-stable form, but is not hypercollapsing.

2. Because of the failure of axiom 2 , theorem 10 also fails for $U_{h c}$-reduction. The term $A$ in the above example has no Böhm $U_{h c}$ tree. (However, Böhm $U_{h c}$ trees, when they exist, are unique. This can still be proved with help of entirely different methods from [KKSdV93].)

\subsection{Comparison of the above notions}

Theorem 33. $U_{h c} \subset U_{r s} \subset U_{\omega} \subset U_{o}$.

\section{References}

[AGM92] S. Abramsky, D. Gabbay, and T. Maibaum, editors. Handbook of Logic in Computer Science, volume II. Oxford University Press, 1992.

[Bar84] H. P. Barendregt. The Lambda Calculus, its Syntax and Semantics. NorthHolland, Amsterdam, 2nd edition, 1984.

[Bar92] H.P. Barendregt. Representing 'undefined' in the lambda calculus. J. Functional Programming, 2:367-374, 1992.

[KKSdV93] J. R. Kennaway, J. W. Klop, M. R. Sleep, and F. J. de Vries. Transfinite reductions in orthogonal term rewriting systems. Technical Report SYS-C93-10, University of East Anglia, Norwich, U.K., 1993. Revised version of [?]. To appear in Information and Computation.

[SPvE93] M. R. Sleep, M. J. Plasmeijer, and M. C. J. D. van Eekelen, editors. Term Graph Rewriting: Theary and Practice. John Wiley, 1993.

[vL90] J. van Leeuwen, editor. Handbook of Theoretical Computer Science, volume B: Formal Models and Semantics. North-Holland, Amsterdam, 1990. 